\title{
¿Cómo formar la ciudadanía global desde entornos virtuales? EI docente frente al trabajo colaborativo en tiempos de pandemia
}

\section{How to form global citizenship from virtual environments? The teacher facing collaborative work in times of pandemic}

Mg. Madeleine Lourdes Palacios Núñez ${ }^{1}$ madeleine.palacios@epg.usil.pe ORCID: https://orcid.org/0000-0001-8050-5946

Dra. Patricia Medina-Zuta² patricia.medina@epg.usil.pe ORCID: https://orcid.org/0000-0002-6315-9356

Dr. C Angel Deroncele-Acosta ${ }^{3}$ angel.deroncele@usil.pe ORCID: https://orcid.org/0000-0002-0413-014X

Fecha de recibo: diciembre 2019, Fecha de aceptación: marzo 2020

\begin{abstract}
Resumen
La pandemia de la Covid-19 trasladó rápidamente las clases a un escenario online, remoto, donde además de los desafíos digitales, también se presenta el reto de seguir formando ciudadanía global en estos entornos virtuales. Por ello, dentro del marco de políticas educativas vigentes, se orienta un análisis reflexivo sobre el proceso formativo y la necesidad de seguir fomentando en y desde el mismo, el aprender a convivir y el aprender a ser, ambos pilares esenciales en la construcción de un ciudadano que priorice el bien común, demostrando autonomía para el aprendizaje y capacidades de autorregulación e interacción fluida con los demás. De esta manera, el trabajo hermenéutico realizado, concluye con la necesidad de una ciudadanía global, que se traduce en la aplicación efectiva de una pedagogía que dinamice el trabajo colaborativo entre los estudiantes y que refuercen el rol reflexivo del docente como guía de este proceso.
\end{abstract}

\footnotetext{
${ }^{1}$ Universidad San Ignacio de Loyola, Lima, Perú

${ }^{2}$ Universidad San Ignacio de Loyola, Lima, Perú

${ }^{3}$ Universidad San Ignacio de Loyola, Lima, Perú
} 


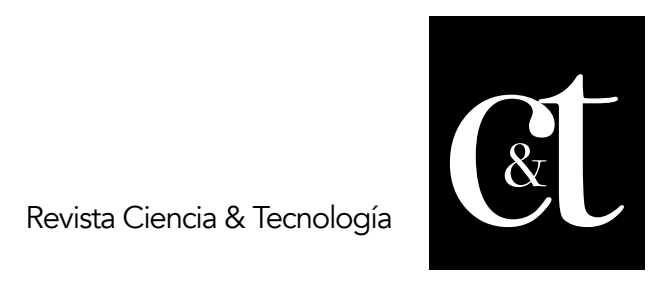

No. 26, 30 de abril de 2020

ISSN impreso: 1390 - 6321

ISSN online: 2661 - 6734

Palabras clave: ciudadanía global, entornos virtuales, trabajo colaborativo, aprender a convivir, aprender a ser.

\begin{abstract}
The Covid-19 pandemic quickly moved classes to an online, remote setting, where in addition to the digital challenges, there is also the challenge of continuing to formation of global citizenship in these virtual environments. Therefore, within the framework of current educational policies, a reflective analysis is oriented on the training process and the need to continue promoting in and from it, learning to live together and learning to be, both essential pillars in the construction of a citizen who prioritizes the common good, demonstrating autonomy for learning and capacities for self-regulation and fluid interaction with others. In this way, the hermeneutical work carried out concludes with the need for global citizenship, which translates into the effective application of a pedagogy that encourages collaborative work among students and that reinforces the reflective role of the teacher as a guide in this process.
\end{abstract}

Keywords: global citizenship, virtual environments, collaborative work, learning to live together, learning to be.

\title{
Introducción
}

Los Objetivos de Desarrollo Sostenible (ODS) presentan de manera relevante al Objetivo de la Calidad Educativa (ODS 4), el cual plantea la necesidad de garantizar una educación inclusiva, equitativa y de calidad, que permita promover oportunidades de aprendizaje perdurable para todos. Este objetivo a su vez, se desglosa en 7 metas; este estudio destaca la séptima, que propone "garantizar un aprendizaje pertinente para el ejercicio de la ciudadanía global" (Comisión Económica para América Latina y el Caribe [CEPAL], 2018).

Frente a esta aspiración de sabernos conducir como ciudadanos del mundo, lo que supone no solo entender el concepto como tal, sino, hacerlo real en nuestra práctica cotidiana, se ha superpuesto una nueva situación, que en la actualidad se traduce como amenaza y como desafío a la vez. Se hace referencia a la pandemia por la Covid-19, que ha traído consigo la consecuente medida de aislamiento social, la cual ha llevado a asumir intempestivamente, procesos formativos absolutamente on-line. Esta sería una condición contextual, en este requisito de forjar la ciudadanía mundial y todo lo que la misma pueda demandar en situaciones de virtualización generalizada.

Desde esta premisa, y asumiendo una postura de docente formador, urge saber entonces, ¿cómo están afrontando la virtualidad los estudiantes? ¿Cómo están conviviendo en este escenario? Probablemente, no se tengan respuestas inmediatas, pero se perfila la intención de seguir fortaleciendo su sentido de pertenencia a la humanidad. Al respecto, la educación remota representa un reto, en tanto existen principios de interacción social como la solidaridad, el diálogo y el respeto hacia la diversidad.

Palacios, Medina, Deroncele. ¿Cómo formar la ciudadanía global desde entornos virtuales? El docente frente al trabajo colaborativo en tiempos de pandemia 


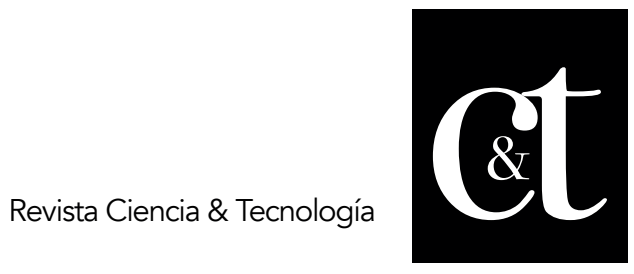

No. 26, 30 de abril de 2020

ISSN impreso: 1390 - 6321

ISSN online: 2661 - 6734

que si bien se siguen manteniendo, demandan ahora mismo, una mayor atención del proceso formativo (Unesco, 2019a), así como atender de manera priorizada las potencialidades de los estudiantes (Deroncele, Medina y Gross, 2020).

Sin embargo, aun cuando el distanciamiento social se ha convertido en una barrera física que impide interactuar de la forma en que estábamos acostumbrados, esto no debería repercutir en este propósito de forjar conciencia de ciudadanía mundial. El entorno virtual en el cual se realizan las clases, representa una oportunidad para poner a prueba la creatividad y la vocación docente, así como la autonomía y el compromiso del estudiante. Esto lleva a reflexionar sobre el proceso de enseñanza y de qué manera podemos potenciar aprendizajes que consoliden capacidades para asumir activamente un rol de ciudadano del mundo.

En esta línea, es relevante lo planteado por la CEPAL (2020), cuando aduce la importancia de poner atención en cuidar del bienestar socioemocional, no solo por esta contingencia; sino porque es esencial que las personas prioricen el bien común como un aspecto fundamental de la convivencia. En este sentido, las mejoras educativas que se implementen necesitarían considerar, aprendizajes que trasciendan a los resultados y al factor meramente cognitivo, para abarcar también, otros aspectos sociales y afectivos.

De esta manera, se busca profundizar en la esencia de este significado de ciudadanía mundial y cómo puede ser viable plantearlo desde el aprendizaje colaborativo, superponiendo el entorno virtual como condición ineludible de la nueva normalidad. Entonces, surgen interrogantes en distintos niveles y focos de atención: ¿qué necesita priorizarse en las políticas educativas mundiales y nacionales para desarrollar una ciudadanía global?, ¿qué aporta el aprendizaje desde sus principales teorías frente a un entorno virtual?, ¿qué será necesario redefinir e incorporar para lograr un rol más eficiente por parte del docente?

\section{Materiales y Métodos}

El artículo centraliza la metodología del análisis documental como un eje central de la aplicación de métodos teóricos basados en procesos de construcción textual argumentativa (Medina y Deroncele, 2019a). Para tal efecto, se han dispuesto algunos procesos de sistematización de fuentes teóricas y contextuales, ampliamente vinculadas a las políticas educativas de orden global y glocal que son fomentadas por organismos científicos representativos. Se complementan a ello, los aportes de investigaciones recientes y de teorías que sustentan las bases de las principales categorías analizadas.

El ejercicio interpretativo constante ha permitido el desarrollo de mecanismos propios del proceso reflexivo sobre la base de un destacable operador epistémico: la problematización (Medina y Deroncele, 2020). Es así, que la síntesis argumentativa implica un proceso complejo, que parte de un sentido heurístico y cuestionador permanente, para arribar poco a poco en la formulación de conclusiones que tienen su soporte en la integración de saberes proporcionados por las distintas fuentes implicadas con la construcción de un sentido lógico y subjetivo de la realidad abordada (Gonzales, 2009).

$$
108
$$




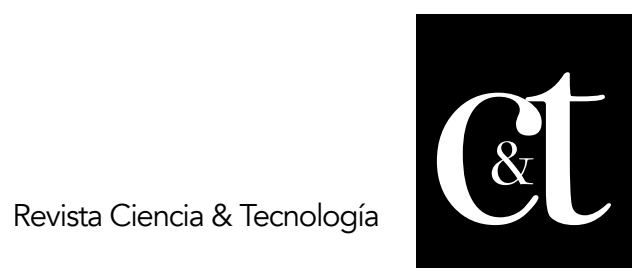

No. 26, 30 de abril de 2020

ISSN impreso: 1390 - 6321

ISSN online: 2661 - 6734

\section{Resultados y Discusión \\ Políticas educativas \\ Aprender a ser y aprender a convivir como pilares de una ciudadanía global}

El marco internacional de políticas educativas ofrece lineamientos que promueven el fortalecimiento de la ciudadanía global. Entre ellos, podemos reconocer el que se plantea en el informe Delors et al. (1996), cuando se mencionan cuatro pilares fundamentales en todo proceso educativo: aprender a conocer, aprender a hacer, aprender a ser y aprender a convivir. De todos ellos, podríamos decir que la educación ha hecho mayor énfasis en los dos primeros, y no se puede ignorar que en la actualidad existe una ruptura del vínculo social, debido a la crisis moral de la violencia por los conflictos interétnicos.

Por ello, urge redoblar nuestra atención hacia aquellos pilares más relevantes para constituir un mayor sentido inter-relacional y de bien común, como el aprender a convivir y el aprender a ser. Estos están estrechamente relacionados, ya que, para aprender a convivir, primero hay que aprender a ser, como consecuentemente, para asumir una ciudadanía mundial, primero es necesario haber aprendido a ser ciudadano de una nación (Mollo y Medina, 2020).

La construcción de una ciudadanía global, demanda asimismo, el desarrollo de una competencia global (OCDE, 2018). Es decir, la capacidad para entender los problemas locales, globales e interculturales; y en modo más concreto, procurar el bienestar común, que tiene su base en saber apreciar y respetar, los diferentes puntos de vista. En palabras de Mollo y Medina (2020), en nuestro país, urge desarrollar el sentido de identidad nacional a través de un enfoque educativo basado en competencias que refuercen la interculturalidad. Asimismo, Medina y Deroncele (2019a) sostienen que la escuela debería fomentar el pensamiento crítico y reflexivo para poder solucionar los problemas que el país necesita. En ese sentido, el reto de la ciudadanía global en la educación no presencial representa todo un desafío, pero también una excelente oportunidad de evolución y de transformación social (Medina y Villalaz, 2020).

La Educación para la Ciudadanía Mundial (ECM) propuesto por Unesco (2019b) tiene como objetivo inculcar en los educandos los conocimientos, habilidades, valores y actitudes necesarios para que construyan un mundo más justo y solidario. Se sostiene así, que el aprendizaje debe basarse en tres ejes integradores: un eje cognitivo para conocer y reflexionar sobre el mundo y sus complejidades; un eje socioemocional para convivir de forma respetuosa y pacífica con los otros, y un eje conductual, para actuar con compromiso en el desempeño desde el rol asumido.

Por su parte, en nuestro país, el Proyecto Educativo Nacional al 2036 - PEN (Consejo Nacional de Educación [CNE], 2020) señala que la tecnología digital conlleva ventajas, pero también riesgos como la "ilusión de la conexión" en el que pueden surgir sentimientos de soledad o limitaciones para la reflexión e introspección personal.

109

Palacios, Medina, Deroncele. ¿Cómo formar la ciudadanía global desde entornos virtuales? El docente frente al trabajo colaborativo en tiempos de pandemia 


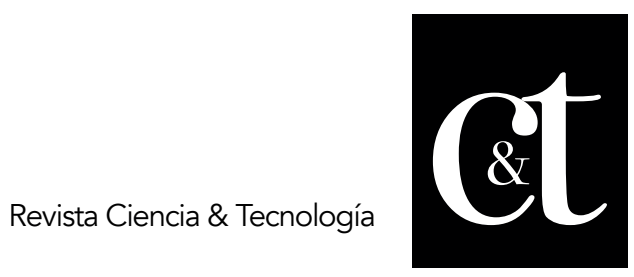

No. 26, 30 de abril de 2020

ISSN impreso: 1390 - 6321

ISSN online: 2661 - 6734

Es así que el PEN orienta sus fines al "reto de la ciudadanía plena", en tanto se enfoca en el bienestar socioemocional en equilibrio con el cognitivo y el físico, porque estos sostienen la base de una práctica ciudadana equilibrada y estable.

En tanto se fomente bienestar y ecuanimidad en el estudiante, será posible que él pueda entender, regular y expresar sus sentimientos, como también a relacionarse e interactuar saludablemente en grupo; incluso en contextos que impliquen desafíos nuevos y complejos como el de la propia pandemia. Este aprendizaje podría replicarse en todos los niveles que necesitan afianzar más de lo humano para el fortalecimiento de capacidades vinculadas a la autonomía, al juicio crítico, a la resolución de problemas, al trabajo en equipo, a la adaptabilidad y al ejercicio de la libertad, entre otros que son piezas determinantes para asumir la ciudadanía global.

Reimers (2015, citado por Medina, 2020), reflexiona sobre la autorregulación e interrelacionamiento como competencias importantes para desarrollar un perfil de graduado ideal del siglo XXI. De allí que el docente que dice asumir su rol formativo plenamente, no deba solamente enfocarse en la dimensión cognitiva y por ende en el saber conocer y el saber hacer.

Lo expuesto ratifica la necesidad de propiciar desde las aulas, las condiciones y experiencias que permitan reforzar aprendizajes relacionados con el ser y el convivir para el desarrollo de la competencia global en el marco de una Educación para la Ciudadanía Mundial. Entonces, cabe reflexionar qué tipo de aprendizaje debemos procurar en los espacios de formación, sobre todo, en contextos virtuales motivados por la pandemia.

\section{La ciudadanía global en entornos virtuales: buscando respuestas desde las teorías del aprendizaje}

A lo largo de la historia, el concepto de aprendizaje ha ido transcurriendo por diferentes corrientes teóricas. La revisión de cada una de estas, permitirá reconstruir una versión del término que contribuya a la comprensión del sentido de una ciudadanía global en las condiciones de un entorno virtual cada vez más apremiante.

Nuestro análisis comienza en el siglo XX con la teoría conductista y algunos de sus principales representantes como Watson. Más adelante estarían Skinner y los neoconductistas, como Bandura. La teoría conductista ha recibido fuertes críticas, pero también ha realizado aportes significativos en la educación. Sin embargo, actualmente existe un rechazo hacia la misma, debido a que la versión primaria de Watson fue concebida como una técnica vinculada a la forma de controlar la conducta del hombre comparado con el de un animal (Bobadilla, 2010).

Entender al ser humano desde esta perspectiva podría ser peligroso, ya que el pretender ejercer control sobre él, implica anular su libertad, su autonomía y su libre albedrío; situación que no estaría contribuyendo con la formación de un ciudadano global que necesita afrontar la realidad cambiante demostrando seguridad y sentido de conciencia como sustento de una postura asumida frente a la vida. 


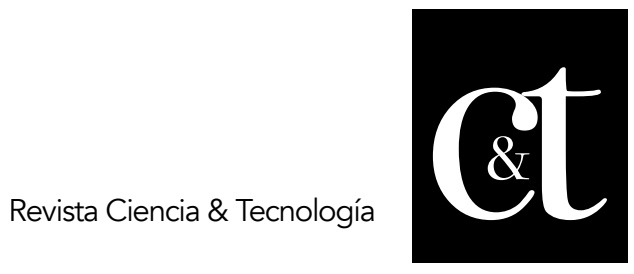

No. 26, 30 de abril de 2020

ISSN impreso: 1390 - 6321

ISSN online: 2661 - 6734

Esta misma objeción a la teoría conductista se complementa, cuando se concibe el condicionamiento clásico de estímulo-respuesta, como un símbolo representativo del aprendizaje como producto de una reacción influenciada por estímulos externos del ambiente.

En el contexto de la educación virtual, sería riesgoso asumir que con solo aplicar la tecnología a la educación ya se logra el aprendizaje, ya que factores internos como la atención y la motivación influyen en el proceso de aprender, en el cual el docente no es un simple operario de las herramientas digitales, y se requiere del mismo, una capacidad reflexiva que le haga examinar y replantear constantemente su práctica. Al respecto, Medina y Deroncele (2019b) destacan un modelo de docente reflexivo y flexible que constantemente revise su propósito de enseñanza, así como el proceso pedagógico en sí mismo; de tal manera que permanente se disponga a evaluarlo y a reajustarlo.

El rol reflexivo del docente es cada vez más trascendente en los contextos formativos e investigativos contemporáneos donde la heterogeneidad y la diversidad es una característica común, signados por las múltiples culturas e historias de vida de los estudiantes (Deroncele, 2015, 2020).

Por otro lado, es mencionable el aporte del condicionamiento operante de Skinner (citado por Jaraba, 2012), el cual puede equipararse a programas computacionales en donde, frente a una respuesta, el estudiante recibe un refuerzo de tipo sonoro, símbolo, etc., que le indica si acertó o pudo emitir una respuesta errada. En ese sentido, en tiempos de clases totalmente online, el quehacer como docente lleva a planificar, diseñar y facilitar este tipo de estímulos y experiencias; que permitan trascender la interacción del estudiante con el ordenador hacia una interacción social entre personas. Así, se reta al docente en el manejo de los recursos digitales para potenciar los procesos de enseñanza con fines formativos.

Posteriormente, con los neoconductistas, surge la teoría del aprendizaje por observación o teoría del aprendizaje social de Bandura, el cual estaría relacionado con el desarrollo de la moral y los valores sociales; más aún si se enfatiza la preocupación que pueden causar los problemas relacionados al incremento de la violencia. En el mismo sentido, Rotter, otro neoconductista, sostiene que la forma de comportarse se aprende en las situaciones sociales; es decir, a través de la mediación de otras personas. Esto también fue llamado aprendizaje vicario, cuyas fuentes de aprendizaje están en el observar y escuchar a otros. Lo anterior es muy importante, siempre que el modelo a imitar sea positivo para que se pueda lograr aprendizajes relevantes para la vida (Bobadilla, 2010). Así, los estudiantes no solo miran al profesor como modelo, sino también a sus propios pares. Sin embargo, ¿cómo podemos propiciar este aprendizaje en los entornos virtuales, en los cuales la interacción física se ha vuelto nula debido al distanciamiento social durante la pandemia?

Sobre la base del Constructivismo, Vigotsky (1978) sostiene que el conocimiento se construye por medio de operaciones y habilidades cognoscitivas en la interacción social, a través de un proceso de transmisión y adquisición de saberes que va, primero, de un plano interpsicológico de interacción y luego a uno intrapsicológico denominado internalización.

Palacios, Medina, Deroncele. ¿Cómo formar la ciudadanía global desde entornos virtuales? El docente frente al trabajo colaborativo en tiempos de pandemia 


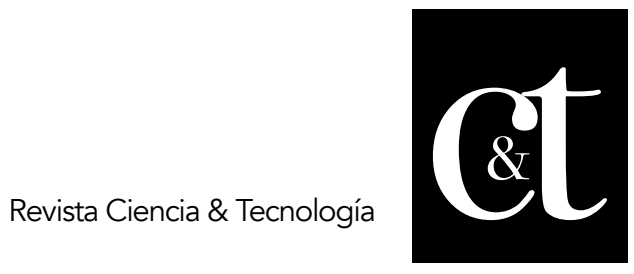

No. 26, 30 de abril de 2020

ISSN impreso: 1390 - 6321

ISSN online: 2661 - 6734

Es decir, la teoría vigotskyana planteaba que lo que un niño puede hacer hoy con la ayuda de alguien en su Zona de Desarrollo Próximo, lo podrá hacer independiente y competente mañana, en su Zona de Desarrollo Potencial.

Bajo la premisa del constructivismo, podemos entonces, identificar experiencias en universidades peruanas que fomentan la interacción social, en la cual el compromiso es mayor cuando aquello que el estudiante construye pueda ser compartido con otros (Fernández-Río, Calderón, Méndez-Giménez y Rolim, 2014). Podemos poner como ejemplo, la posibilidad de que los estudiantes de un curso de redacción, elaboren en equipo una revista digital en genial-ly o flipsnack que generalmente, contiene textos expositivos o argumentativos sobre temas de interés local, regional o mundial. Otro ejemplo, podría ser, en un curso de desarrollo personal y social, cuando los estudiantes elaboran de forma colaborativa una página web en wixsite o wordpress que contiene tips para mejorar sus competencias socioemocionales. Esta producción de materiales autoconstruidos culmina con la etapa de la socialización, en la cual no solo comparten el conocimiento aprendido, sino que también, reciben comentarios, a modo de retroalimentación.

De esta manera, el estudiante en la era digital no solo es consumidor de información, sino que también la produce, lo que actualmente se ha denominado como "prosumidor", refiriéndose a una persona capaz de aprovechar lo que le ofrece la tecnología para generar su propio contenido digital (Llaullipoma et al., 2019).

Esto representa una excelente oportunidad para fortalecer la ciudadanía global siempre y cuando se oriente a la investigación y discusión sobre temas de impacto (asuntos de índole global y local), y se promueva, a través de este tipo de experiencias, competencias socioemocionales en el estudiante como el trabajo en equipo, el pensamiento crítico, la autonomía y responsabilidad, la comunicación y colaboración, la empatía, entre otros. En esta intención, se hace necesario que el docente no descuide su rol de guía y tutor en el proceso de diseño de la experiencia colaborativa.

Hoy en día, se habla del aprendizaje basado en competencias que integra tres áreas: cognitiva, procedimental y actitudinal. De esta manera, la educación se vuelve integradora y compleja, pero cabe preguntarnos qué significa ser complejo. Al respecto, Morin (1999) se refiere a la complejidad como "una diversidad propiamente biológica en el seno de la unidad humana" (p.27). Es decir, el ser humano es complejo en tanto, no solo es racional (sapiens), sino también está conformado por otras dimensiones como delirio (demens), trabajo (faber), juego (ludens), empírismo (empiricus) e imaginación (imaginarius), entre los más relevantes. Entender esta complejidad del ser humano (homo complexus), debería ser la base sobre la cual se fundamente el nuevo enfoque para la educación de la era digital, de manera que no se centre solo en lo académico o técnico, sino que trascienda en la formación del educando en aspectos que abarquen lo actitudinal, afectivo y social.

A partir de lo expuesto, consideramos que, para poder contribuir con la formación de la ciudadanía global desde los entornos virtuales, se necesita fortalecer el pilar del aprender a ser y el aprender a convivir. 


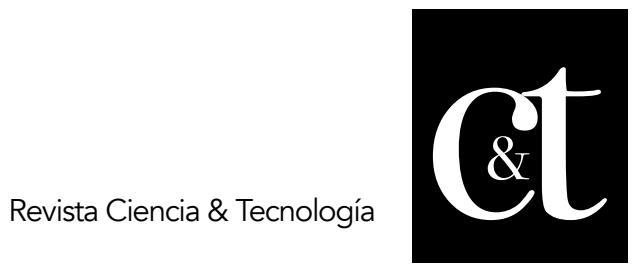

No. 26, 30 de abril de 2020

ISSN impreso: 1390 - 6321

ISSN online: 2661 - 6734

Respecto a este último, el trabajo colaborativo surge como una propuesta de suma pertinencia, en tanto favorece el aprendizaje autónomo del estudiante y también lo desafía a trabajar en interacción con otros, aun cuando no haya oportunidad de contacto físico, sino virtual. Al respecto, Mollo y Medina (2020) sostienen que el trabajo en equipo enseña a "aprender a convivir" en escenarios complejos como el que la educación no presencial estaría demandando.

El trabajo colaborativo: pieza clave de la ciudadanía global desde la acción docente Ante la necesidad de fortalecer aprendizajes relevantes para la vida como aprender a ser y aprender a convivir, consideramos que el tipo de experiencias más pertinente es la del trabajo colaborativo en entornos virtuales.

Para Johnson y Johnson (citados por Guerra, Rodríguez y Artiles, 2019), el trabajo colaborativo se basa en la cooperación y significa trabajar juntos para alcanzar objetivos comunes. Asimismo, la implementación del mismo, contribuye al desarrollo de cinco dimensiones esenciales: "la interdependencia positiva, la promoción a la interacción, la responsabilidad individual, las habilidades y las destrezas de trabajo grupales y la interacción positiva". Si vinculamos lo que sostiene el autor y lo que plantea Delors y otros (1996), encontraremos que el aprender a ser y el aprender a convivir sostienen las cinco dimensiones del trabajo colaborativo, ya que destacan aspectos como la interacción, la responsabilidad, la interdependencia y el trabajo en colectivo interactivo.

En tiempos de pandemia, el trabajo colaborativo mediado por la tecnología recibe un impacto que puede ser beneficioso si se canaliza adecuadamente. Diversas experiencias van dando cuenta de este aporte. Al respecto, Caballero-González y García-Valcárcel (2020) concluyeron que el aprendizaje con robótica educativa en la escuela contribuye a fortalecer el pensamiento computacional pero también habilidades intrapersonales como la creatividad y la regulación de la conducta, así como las habilidades interpersonales ligadas a la colaboración, comunicación y a la construcción de una comunidad. En la misma lógica, Corujo, Gómez y Merla (2020), propusieron el empleo del Webquest como estrategia de aprendizaje para reforzar la competencia didáctica en los futuros maestros y también otras competencias más transversales como el trabajo en equipo y el aprendizaje autónomo.

Sin embargo, el éxito en estas experiencias no se da en automático. Al respecto, Younie, Leask, y Burden (2015) sostienen que, a pesar de la importancia de las TICS, estas no tienen un impacto en el aprendizaje por sí solas, sino que dependen del uso pedagógico con que sean incorporadas por el docente. Según Guitert y Romeu (2019), los roles del docente en línea pueden ir direccionando una configuración de diseñador, gestor, guía/acompañante, dinamizador, evaluador, colaborador e investigador. De allí que el ser docente no estaría siendo limitado a asumir un rol en el propio desarrollo de las clases virtuales, sino en la gestión y previsión de las mismas, de la misma manera como lo hacía cuando las clases eran presenciales. Aunque ello, estaría implicando poner atención a la previsión de actividades sincrónicas y asincrónicas que configuren procesos pedagógicos más pertinentes al contexto de una educación remota.

Palacios, Medina, Deroncele. ¿Cómo formar la ciudadanía global desde entornos virtuales? El docente frente al trabajo colaborativo en tiempos de pandemia 


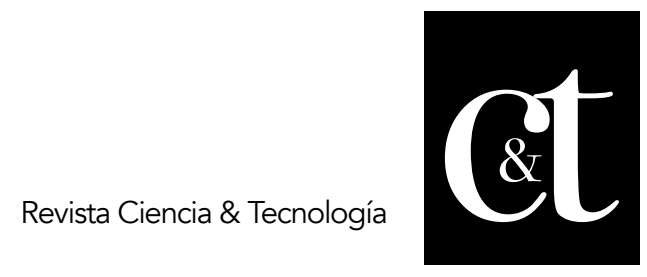

No. 26, 30 de abril de 2020

ISSN impreso: 1390 - 6321

ISSN online: 2661 - 6734

Sin embargo, aun cuando muchas instituciones de educación superior han llevado a cabo grandes esfuerzos por capacitar a los docentes en el uso y manejo de las herramientas digitales, se ha omitido o abordado de forma poco profunda la actualización del docente respecto al uso estratégico de las Nuevas Tecnologías de la Información y Comunicación (NTIC), Tecnologías de Aprendizaje y Conocimiento (TAC) y, sobre todo, de las Tecnologías para el Empoderamiento y la Participación (TEP). Todas ellas muy útiles para propiciar la interacción y la colaboración en relación a la creación de contenido dentro de una comunidad virtual (Ruíz, 2014).

En ese sentido, más que la dimensión tecnológica por sí misma, lo que se necesita del docente es que pueda incidir más en aplicar una pedagogía digital basada en la constitución de experiencias efectivas de aprendizaje en entornos virtuales. En la investigación realizada por Cebreiro (2017), se constató que muchos docentes no estaban favoreciendo la colaboración y el aprendizaje social durante experiencias educativas virtuales. Frente a ello, se les recomendó combinar el aprendizaje formal con el informal. Esto suponía incorporar el concepto de una educación más "vernacular" que integre por ejemplo, redes sociales como Facebook, Twitter, Instagram, entre otras. Se estaría favoreciendo con ello una mayor motivación e interés por parte del estudiante. Asimismo, se señaló que el acompañamiento y el seguimiento se habrían mellado con la no presencialidad, pero que poco se hacía para suplir estas carencias. A decir de los propios docentes que participaron en estos estudios, estas situaciones podrían tener su explicación en la alta importancia que se le daba a los tecnicismos sobre cómo escribir una programación didáctica (estructura, formas verbales), en contraposición con una baja atención al cómo enseñar a distancia cuando se realizaban los programas de capacitación y perfeccionamiento docente.

Relacionando la acción docente con el trabajo colaborativo, Johnson y Johnson (citados por Guerra, Rodríguez y Artiles, 2019), sostienen que debe haber un cambio importante en su rol. Ahora su papel será de "guía, gestor y organizador del aprendizaje, fomentando siempre la autonomía de sus alumnos/as". En otros términos, el docente no es un simple transmisor de conocimiento u operario de dispositivos tecnológicos, sino que es aquel que propicia las condiciones para que los estudiantes, en su interacción social, puedan construir el conocimiento, siempre con un acompañamiento que no reste autonomía.

Respecto al término de competencia digital y en el marco de competencias de los docentes en materia de TIC determinado por la Unesco (2019c), se señalan 18 competencias digitales que se obtienen de cruzar los seis objetivos de la práctica profesional de los docentes con las tres etapas o niveles de desarrollo en que se organiza respecto al uso pedagógico de las TIC. Se observa que, en el primer nivel denominado adquisición de conocimientos, el logro implica demostrar manejo de los recursos tecnológicos en las clases, luego en un segundo nivel se denota el conocimiento y destreza en el uso de las TIC respecto a la resolución de conflictos. Esto supone acciones específicas como el saber implementar metodologías emergentes a partir de proyectos colaborativos, que potencien la comunicación, la reflexión crítica y la expresión creativa. Finalmente, en un tercer nivel se determina la capacidad creativa del docente que implica crear conocimientos a partir de una pedagogía que dinamice actividades colaborativas entre estudiantes que den paso a aptitudes interpersonales y de autorregulación. 


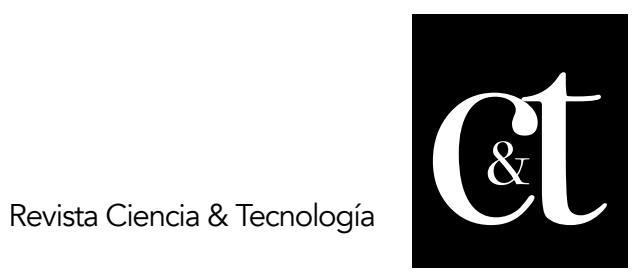

No. 26,30 de abril de 2020

ISSN impreso: 1390 - 6321

ISSN online: 2661 - 6734

Frente a ello, la Dirección de Innovación Tecnológica en Educación del gobierno peruano (Minedu/DITE) formuló un perfil de competencia digital docente acorde a las necesidades del país respondiendo al Marco de Buen Desempeño Docente (MBDD). En el mismo se orientan el logro de siete desempeños: (1) gestión de la información académica, (2) gestión de Información, (3) participación en entornos digitales colaborativos, (4) diseña actividades de aprendizaje, (5) conducción de la experiencia de aprendizaje, (6) evaluación del proceso y logro de los aprendizajes e (7) incorporación de entornos virtuales como recurso y estrategia para el aprendizaje (Sulmont, 2017). Como se puede apreciar, la colaboración sería parte de la agenda educativa peruana. Sin embargo, todavía se hace necesario impulsar acciones concretas que permitan formar al docente como un líder capaz de generar cambios y de propulsar una educación de calidad (Alemán, Medina y Deroncele, 2020), sobre todo, en contextos retadores como el de la educación virtual.

Si el trabajo colaborativo en línea no es debidamente orientado, en lugar de afianzar el sentido de pertenencia a un grupo humano, generaría el rechazo al ser y por ende al convivir, lo cual retrasaría la construcción de actitudes y disposiciones propias de una ciudadanía global. Sobre el punto, Capdeferro y Romero (2012) identificaron que, cuando se trabaja de forma colaborativa en línea, las fuentes de frustración de un mayor número de personas, se centran en el desequilibrio, en el nivel de compromiso, en la responsabilidad y en el esfuerzo entre los integrantes que conforman un grupo de trabajo. Así también se verían afectadas sus habilidades de negociación para resolver conflictos. En esta línea, también, se mencionó que el proceso evaluativo, generalmente estaría enfocado en el resultado final y no en aspectos de desarrollo más suscritos a las participaciones y disposiciones personales. Una fuente de frustración, poco explorada, sería la del instructor, quien -según el $10 \%$ de estudiantes- no interactuó con el grupo y no brindó orientación oportuna o una acción correctiva al tomar consciencia de un determinado problema. Los estudiantes esperaban retroalimentación oportuna por parte del docente.

Respecto a los obstáculos para el trabajo colaborativo efectivo, Le, Janssen y Wubbels (2018), hallaron que los docentes generalmente suelen enfocarse en las metas académicas más que en las metas colaborativas. Esto implica que los docentes no disponen en los estudiantes el desarrollo de habilidades sociales y colaborativas como la asignación de roles, el escucharse atentamente unos y otros y el aceptar diferentes puntos de vista. Los docentes mencionaron que gran parte del problema radicaba en la presión que tenían al exigírseles el desarrollo de mucho contenido y que muchas veces el mismo, no estaba contemplado en el plan de estudios. Además, no se sentían que estaban capacitados para entrenar en habilidades colaborativas al conjunto de sus estudiantes.

Todo ello permite pensar que se necesita disponer experiencias de aprendizaje colaborativo en línea, en las cuales el docente denote una práctica reflexiva y sea capaz de examinar y dilucidar las limitaciones y desventajas que acarrea la no presencialidad. Por lo tanto, debe desempeñar un rol proactivo en el seguimiento de las actividades programadas; asegurándose de que el grupo se orienta a la acción colaborativa. En complemento a ello, no basta con hacer énfasis solo en los aspectos de la didáctica y de la metodología; se requiere un docente fortalecido en sus habilidades blandas y competencias socioemocionales, que pueda brindar soporte orientación a sus estudiantes.

$$
115
$$

Palacios, Medina, Deroncele. ¿Cómo formar la ciudadanía global desde entornos virtuales? El docente frente al trabajo colaborativo en tiempos de pandemia 


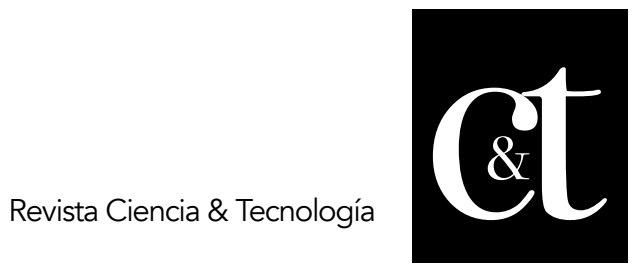

No. 26, 30 de abril de 2020

ISSN impreso: 1390 - 6321

ISSN online: 2661 - 6734

Esteve, Castañeda y Adell (2018) presentan un Modelo Holístico de Competencia Docente para el Mundo Digital, en el cual el docente asume su compromiso social de integración de las TICS al proceso formativo desde un rol práctico y reflexivo. En este modelo, las experiencias de aprendizaje son enriquecedoras, en tanto el docente revisa permanentemente su discurso pedagógico y se propone transformaciones para optimizar el proceso de enseñanza-aprendizaje (Medina y Deroncele, 2019b).

Se trata de forjar un docente con gran expertis en el manejo de habilidades sociales como la empatía, la solidaridad y la comunicación (Tapia-Gutiérrez y Cubo-Delgado, 2017). En el webinar realizado por Unesco en el Perú (3 de junio del 2020), Patricia Cabrerizo señaló que es importante primero que el docente desarrolle esta competencia en el vínculo con sus pares para que luego pueda proyectarla hacia sus estudiantes. Asimismo, Martín Vegas, coordinador del programa Horizontes de Unesco Perú y ex Vice Ministro de Educación, afirmó que el reto no es estar contactado con el estudiante, sino conectado de forma periódica con un "cómo estás", mostrar tu rostro, aunque sea un breve tiempo, escuchar y sentir. Así, la crisis de la pandemia es una oportunidad para buscar y encontrar nuevas formas de relacionarnos empáticamente.

Sin embargo, el afán de trasladar este propósito a la práctica formativa en Perú, requiere plantearse si realmente el docente y el sistema educativo peruano están preparados para implementar el trabajo colaborativo en entornos virtuales. Si bien, todavía hay mucho que examinar al respecto, también existen algunas alternativas que pueden ser viabilizadas en la consecución de este ideal de forjar ciudadanos globales desde las actuales y las nuevas generaciones.

\section{Conclusiones}

1. Urge comprometernos con una educación para la ciudadanía mundial en la que se refuercen el aprender a ser y aprender a convivir y se cuide el bienestar socioemocional para lograr la competencia global, sobre todo, en escenarios complejos como los entornos virtuales.

2. El concepto de aprendizaje ha ido evolucionando a través del tiempo y es relevante el aporte que brindan las teorías del aprendizaje. En este proceso, se ha perfilado el rol del docente como facilitador y guía de experiencias de aprendizaje pertinente, así como el papel cada vez más autónomo del estudiante, pero en interacción con los demás para lograr aprendizajes relevantes.

3. El trabajo colaborativo es una propuesta muy conveniente como metodología en entornos virtuales, ya que refuerza habilidades intrapersonales e interpersonales como la creatividad, la comunicación, la colaboración y la empatía. Todas ellas tan necesarias para aprender a convivir y aprender a ser en el marco de una ciudadanía global.

4. El fortalecimiento del rol docente necesita integrar el desarrollo del trabajo colaborativo en entornos virtuales, a fin de contribuir con una visión estratégica de los procesos de aprendizaje. De allí que se requiere una formación del profesorado universitario que trascienda lo técnico de la competencia digital y aterrice en un modelo integral y complejo que amalgame lo tecnológico, lo pedagógico y lo socioemocional. 


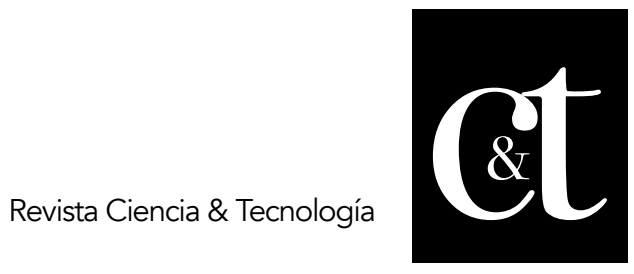

No. 26, 30 de abril de 2020

ISSN impreso: 1390 - 6321

ISSN online: 2661 - 6734

\section{Referencias}

Alemán-Saravia, A.C., Medina-Zuta, P. y Deroncele-Acosta, A. (2020). La calidad docente en un marco de equidad: balance de las políticas educativas y su contextualización en la realidad peruana. Maestro y Sociedad, 17(4), 762-782. Recuperado de: https://maestroysociedad.uo.edu.cu/index.php/MyS/article/view/5243

Bobadilla, D. (2010). El conductismo: orígenes, trayectoria y significado. Universidad de San Martín de Porres. Recuperado de: https://cutt.ly/Zfz70Fd

Caballero-González, Y. A. y García-Valcárcel, A. (2020). Fortaleciendo el pensamiento computacional y habilidades sociales mediante actividades de aprendizaje con robótica educativa en niveles escolares iniciales. Pixel-Bit. Revista de Medios y Educación, 58, 117-142. Recuperado de: https://doi.org/10.12795/pixelbit.75059

Capdeferro, N. \& Romero, M. (2012). Are Online Learners Frustrated with Collaborative Learning Experiences? [¿Los estudiantes en línea se sienten frustrados con las experiencias de aprendizaje colaborativo?]. The International Review of Research in Open and Distributed Learning, 13 (2), 26-44. Recuperado de: https://doi.org/10.19173/irrodl.v13i2.1127

Cebreiro, B., Fernández, C. y Arribi, J. (2017). Formación profesional a distancia: corriendo en la dirección equivocada. Píxel-Bit. Revista de Medios y Educación, 50, 65-76. Recuperado de: http://dx.doi.org/10.12795/pixelbit.2017.i50.04

Comisión Económica para América Latina y el Caribe [CEPAL - Unesco] (2018). La Agenda 2030 y los objetivos de desarrollo sostenible. Naciones Unidas. Recuperado de: https://repositorio.cepal.org/bitstream/handle/11362/40155/24/S1801141 es.pdf

Comisión Económica para América Latina y el Caribe CEPAL - Unesco (2020). La educación en tiempos de la pandemia de Covid-19. Recuperado de: https://repositorio.cepal.org/bitstream/handle/11362/45904/1/S2000510 es.pdf

Consejo Nacional de Educación [CNE]. (2019). Proyecto Educativo Nacional al 2036. Recuperado de: http://www.cne.gob.pe/uploads/publicaciones/boletin/revista-cnefinal-n-44.pdf

Corujo, M. C, Gómez, M.T, \& Merla, A. E. (2020). Construtivist and collaborative methodology mediated by ict in Higher education. [Constructivismo y metodología colaborativa mediada por TIC en educación superior usando webquest]. Pixe/Bit.Revista de Medios y Educación, 57, 7-57. Recuperado de: https://doi.org/10.12795/pixelbit.2020.i57.01

Delors, J., Al Mufti, I., Amagi, I., Carneiro, R., Chung, F., Geremek, B., \& Nazhao, Z. (1996). Informe a la Unesco de la comisión internacional sobre la educación para el siglo XXI: La educación encierra un tesoro. Madrid: Santillana, Ediciones UNESCO.

Deroncele, A. (2020). Paradigmas de investigación científica. Abordaje desde la competencia epistémica del investigador. Revista Arrancada, 20(37), 211-225. Recuperado de: https://revistarrancada.cujae.edu.cu/index.php/arrancada/article/download/331/233

Deroncele, A., Medina, P., y Gross, R. (2020). Gestión de potencialidades formativas en la persona: reflexión epistémica y pautas metodológicas. Universidad y Sociedad, 12(1), 97-104. Recuperado de: https://rus.ucf.edu.cu/index.php/rus/article/view/1417

117

Palacios, Medina, Deroncele. ¿Cómo formar la ciudadanía global desde entornos virtuales? El docente frente al trabajo colaborativo en tiempos de pandemia 


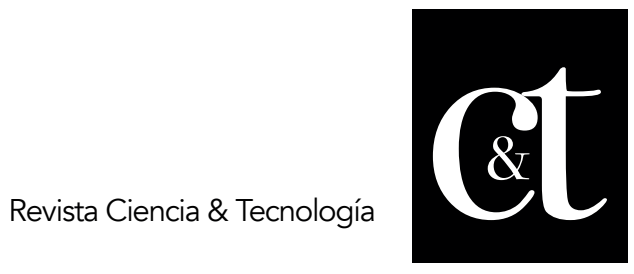

No. 26,30 de abril de 2020

ISSN impreso: 1390 - 6321

ISSN online: 2661 - 6734

Deroncele, A. (2015). Estrategia educativa para la formación profesional integral del psicólogo en el contexto organizacional. Tesis de doctorado. Recuperado de: https://cutt.ly/xtwj6pp

Esteve, F., Castañeda, L., y Adell, J. (2018). Un Modelo Holístico de Competencia Docente para el Mundo Digital. Revista Interuniversitaria de Formación del Profesorado, 32(1), 1-13. Recuperado https://www.redalyc.org/jatsRepo/274/27454937017/index.html

Fernández-Río, J., Calderón, A., Méndez-Giménez, A., y Rolim, R. (2014). Teoría construccionista del aprendizaje en formación del profesorado. Perspectivas de alumnado y profesorado desde la investigación cuantitativa y cualitativa. Profesorado. Revista de currículum y formación del profesorado, 18(3), 214-228. Recuperado de: https://dialnet.unirioja.es/servlet/articulo?codigo $=5006947$

Gonzales, F. (2007). Investigación cualitativa y subjetividad: Los procesos de construcción de la información. México D.F.: McGraw-Hill.

Guerra, M, Rodríguez, J., y Artiles, J. (2019). Aprendizaje colaborativo: experiencia innovadora en el alumnado universitario. REXE. Revista de Estudios y Experiencias en Educación, 18(36), Recuperado de: https://doi.org/10.21703/rexe.20191836guerra5

Guitert, M. y Romeu, T. (2019). Estrategias para la docencia en línea. Universitat Oberta de Catalunya. FUOC

Jaraba, R. (2012). Psicología del aprendizaje. Corporación Universitaria del Caribe. Recuperado de: https://cutt.ly/xfz75wm

Largo, V. (2016). El problema del indígena en Manuel González Prada. OGIGIA. Revista electrónica de estudios hispánicos, 19, 41-56. Recuperado de: https://dialnet.unirioja.es/descarga/articulo/6301222.pdf

Llaullipoma, J. A., Mendoza, G., Hernández, A., Hinostroza, S. y Roman, D. (2019). Motivando la formación de docentes prosumer: una experiencia de estudiantes de educación de una universidad peruana. En Pontifica Universidad Católica del Perú, XXII Congreso Internacional Tecnología e innovación para la diversidad y calidad de Ios aprendizajes. Recuperado de: https://cutt.ly/jhcfbUR

Medina, P., y Deroncele, A. (2020). La práctica investigativa dialógico-reflexiva para orientar la problematización como operador epistémico de la construcción científicotextual. Revista Inclusiones, 7(2), 37-46. Recuperado de: https://cutt.ly/AhcfE47

Medina, P., y Deroncele, A. (2019a). La construcción científico-textual en el posgrado: el desafío de la transdisciplinariedad y la reflexividad. Maestro y Sociedad, 16(4), 829838. Recuperado

de: https://maestroysociedad.uo.edu.cu/index.php/MyS/article/view/5015

Medina, P., y Deroncele, A. (2019b). La evaluación formativa desde el rol del docente reflexivo. Maestro $y$ Sociedad, 16(3), 597-610.Recuperado de: https://maestroysociedad.uo.edu.cu/index.php/MyS/article/view/4979

Medina, P. (2020, 5 de junio). ¿Cómo enfrentamos los procesos de enseñanza y aprendizaje en la educación no presencial? [conferencia]. Primer Encuentro de experiencias formativas en tiempos de pandemia de la Red KIPUS Perú. https://www.redkipusperu.org/inicio/primer-encuentro-de-experiencias-formativasen-tiempos-de-pandemia-de-la-red-kipus-peru/

$$
118
$$

Revista científica Ciencia y Tecnología Vol 20 No 26 págs. 106-119

http://cienciaytecnologia.uteg.edu.ec 


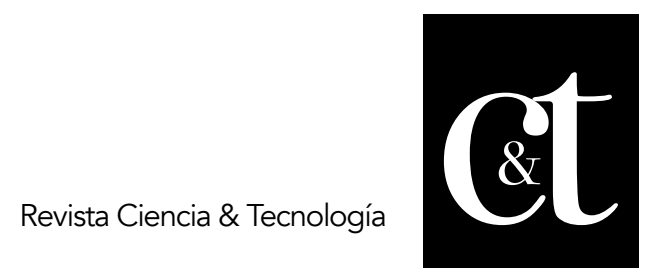

No. 26, 30 de abril de 2020

ISSN impreso: 1390 - 6321

ISSN online: 2661 - 6734

Ministerio de Educación Cultura y Deporte de España [MECD]. (2018). Marco de competencia global. Estudio PISA. Recuperado de: https://sede.educacion.gob.es/publiventa/d/22445/19/00

Morin, E. (1999). Los siete saberes necesarios para la educación del futuro. Organización de las Naciones Unidas para la Educación, la Ciencia y la Cultura. Recuperado de: https://cutt.ly/lfz75vg

Mollo, M., y Medina, P. (2020)._La evaluación formativa: hacia una propuesta pedagógica integral en tiempos de pandemia. Maestro y Sociedad, 17(4), 635-651. Recuperado de: https://maestroysociedad.uo.edu.cu/index.php/MyS/article/view/5235

Organización de las Naciones Unidas para la Educación, la Ciencia y la Cultura [Unesco]. (2015). Educación para la Ciudadanía Mundial. Recuperado de: https://unesdoc.unesco.org/ark:/48223/pf0000233876

Organización de las Naciones Unidas para la Educación, la Ciencia y la Cultura [Unesco]. (2017). Una mirada a la profesión docente en el Perú: Futuros docentes, docentes en servicio y formadores de docentes. Ministerio de Educación del Perú. Recuperado de: https://es.unesco.org/themes/ecm/definicion

Organización de las Naciones Unidas para la Educación, la Ciencia y la Cultura Perú [Unesco] (2019a). Educación para la Ciudadanía Mundial: para un enfoque global. Recuperado de: https://es.unesco.org/news/educacion-ciudadania-mundialenfoque-global

Organización de las Naciones Unidas para la Educación, la Ciencia y la Cultura [Unesco] (2019b). ¿En qué consiste la Educación para la Ciudadanía Mundial? Recuperado de: https://es.unesco.org/themes/ecm/definicion

Organización de las Naciones Unidas para la Educación, la Ciencia y la Cultura [Unesco] (2019c), Marco de competencias de los docentes en materia de TIC, París [en línea]. Recuperado de: https://cutt.ly/ghcfJPb

Organización de las Naciones Unidas para la Educación, la Ciencia y la Cultura Perú [Unesco] (2020, 3 de junio). ¿Por qué es importante la empatía docente en la crisis actual? [Webinar]. Recuperado de:https://cutt.ly/Cfz760K

Organización para la Cooperación y el Desarrollo Económicos. (2018). Preparing our youth for an inclusive and sustainable world. Recuperado de: https://www.oecd.org/education/Globalcompetency-for-an-inclusive-world.pdf

Sulmont, L. (2017). Definición de la Competencia Digital Docente y propuesta de formación. Recuperado de: https://es.slideshare.net/leasulmont/cultura-digital-2017-cddstorytelling-lea-sulmont

Tapia-Gutiérrez, C. y Cubo-Delgado, S. (2017). Habilidades sociales relevantes: percepciones de múltiples actores educativos. magis, Revista Internacional de Investigación en Educación, 9 (19), 133-148. doi: 10.11144/Javeriana.m9-19.hsrp

Villalaz-Castro, E. S., y Medina-Zuta, P. (2020). El currículo universitario peruano: aspectos complejos. Maestro y Sociedad, 17(1), 121-136. Recuperado de: http://www.maestroysociedad.uo.edu.cu

Younie, S., Leask, M., \& Burden, K. (2015). Teach and learning with ICT in the primary school [Enseñar y aprender con las TIC en la escuela primaria]. Routledge. Recuperdo de: https://cutt.ly/ufz76IF

Palacios, Medina, Deroncele. ¿Cómo formar la ciudadanía global desde entornos virtuales? El docente frente al trabajo colaborativo en tiempos de pandemia 